(C) 2009 IEEE. Personal use of this material is permitted. Permission from IEEE must be obtained for all other uses, in any current or future media, including reprinting/republishing this material for advertising or promotional purposes, creating new collective works, for resale or redistribution to servers or lists, or reuse of any copyrighted component of this work in other works. 


\title{
Joint Channel and Phase Noise Compensation for OFDM in Fast-Fading Multipath Applications
}

\author{
Roberto Corvaja, Member, IEEE, and Ana García Armada, Senior Member, IEEE
}

\begin{abstract}
Orthogonal frequency-division multiplexing (OFDM) systems, such as the new wireless standards or the schemes proposed for third-generation (3G) evolution, exhibit great sensitivity to the effects of phase noise and the time-varying propagation channel, which can introduce interchannel interference (ICI) due to the loss of orthogonality among subcarriers. In this paper, joint channel estimation and ICI reduction schemes are investigated, which compensate the effects of phase noise and multipath channel in a realistic scenario, where the channel is not assumed perfectly known, whereas its estimation is obtained by combining a decision-feedback scheme and a pilot-aided estimator. We propose a technique for ICI compensation that has reduced complexity by only considering the most significant terms of ICI.
\end{abstract}

Index Terms-Channel estimation, interchannel interference (ICI), orthogonal frequency-division multiplexing (OFDM) systems, phase noise.

\section{INTRODUCTION}

$\mathbf{O}$ RTHOGONAL frequency-division multiplexing (OFDM) systems are already being employed in wireless networks for fi ed and low-mobility environments, such as wireless local area networks (WLAN) or wireless metropolitan area networks (WMAN), and are considered to be candidates for the next generation of mobile communications for their $\mathrm{fl}$ xibility, ease of implementation, and equalization [1], [2]. In particular, the Third-Generation Partnership Project (3GPP) proposal for the long-term evolution (LTE) of Universal Mobile Telecommunications System (UMTS) again considers OFDM but with many more subcarriers than in WLAN. However, the use of OFDM in a mobile environment also gives rise to a degradation due to the changing channel, together with the effect of the phase noise. The performance of OFDM systems in the presence of phase noise has been analyzed in several works [3]-[7]. Some schemes to estimate and compensate the common phase error (CPE) arising from the phase noise in OFDM are presented in [6], [8], and [9]. In these works, the propagation channel is assumed perfectly known. In [7], a dual-branch estimation is proposed, with an unmodulated version of the phase noise

This work was supported in part by Project "MACAWI" under Grant TEC2005-07477-C02-02 and by Project "MAMBO 2" under Grant CCG06-UC3M-TIC-0698.

R. Corvaja is with the Department of Information Engineering, University of Padova, Padova, Italy.

A. García Armada is with the Department of Signal Theory and Communications, University Carlos III of Madrid, Leganés, Spain. coming from the receiver oscillator. To account for the effect of the interchannel interference (ICI) due to phase noise, beyond the CPE term, a minimum mean square error (MMSE) percarrier equalization is considered in [8], [10], and [11], modeling the ICI as an equivalent additive noise. This requires the estimation of the ICI variance, which has also been addressed in [12].

Other schemes to compensate more ICI coefficient are considered in [9] and [13]. However, in all these ICI compensation schemes, channel estimation is considered ideal, which is diffi cult to accomplish in a practical implementation.

In [14], the propagation channel is not considered known, and a pilot-aided scheme is proposed to compensate the ICI deriving from Doppler in a fast-fading channel in the absence of phase noise. Linearization of the channel evolution is used to derive a closed-form expression for the signal-to-interference ratio (SIR).

In a previous work of one of the authors of this paper [15], a block equalization of the phase noise on the whole OFDM symbol is proposed, leading to a better performance than a percarrier equalization scheme. Again, the channel is considered ideally estimated. Here, we extend this paper by removing the assumption of perfect channel knowledge separate from the phase noise process, and we jointly consider the effect of phase noise and the channel, proposing a practical equalization scheme to reduce the ICI effects. To the best of our knowledge, schemes that compensate both phase noise and channel effects have not yet been proposed. In detail, we develop a joint channel equalization and phase-noise compensation scheme, which reduces the ICI components, by considering an equivalent channel matrix that comprises the effects of both the phase noise and the fading channel. By increasing the accuracy in the matrix representation, better results are obtained at the expense of an increasing complexity.

The application of the proposed schemes to practical systems, such as LTE and IEEE 802.16 (Wimax), is then considered to present some numerical results.

In addition to the effects considered in this paper, the timevarying channel introduces a random frequency offset within the Doppler spread range. Together with the frequency offset due to the mismatch between the receiver and transmitter oscillators, they represent another source of ICI. It is known that in OFDM, as the subcarriers are closely spaced over the channel bandwidth, the frequency offset must be kept within a small fraction of the subcarrier spacing to avoid severe bit error rate degradations. The problem is solved in the receiver by the accurate synchronization of the carrier frequency [16]-[18] prior to detection. Therefore, although these 


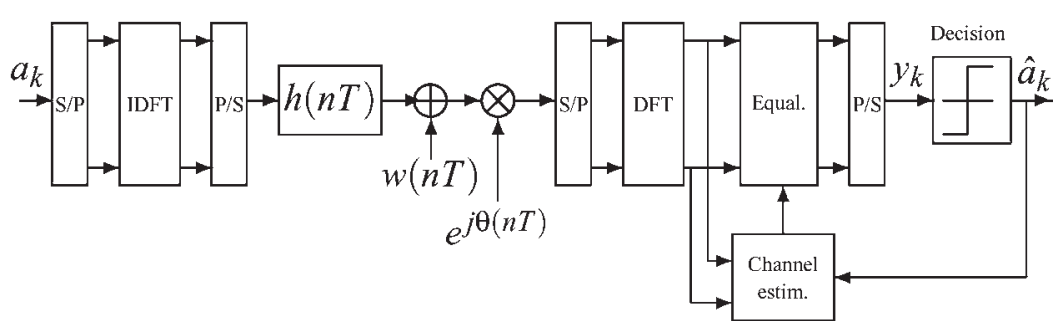

Fig. 1. Scheme of the OFDM transmission system.

frequency-synchronization algorithms may leave a residual offset, the ICI produced is much lower than the effects that we compensate in this paper.

The ICI cancellation schemes proposed and evaluated here require an equivalent channel matrix, which is estimated by an optimum combination of a pilot-based and decision-feedback technique. We assume that the transmission is organized in a frame of several OFDM symbols, where possibly one or more of them are transmitted as a preamble at the beginning of a frame. This applies, for example, to WLAN and WMAN systems, such as the standards IEEE 802.11 (WiFi) or IEEE 802.16 (Wimax). In LTE, only scattered pilot subcarriers are present, which are optimum for the mobile channel, rather than using preambles [19]. Therefore, for LTE, we cannot consider a whole OFDM symbol per frame to be completely known, but the techniques proposed here can also be applied in the absence of a known preamble, as we will show. Since, together with the Doppler effect, the result of the phase noise is comparable to a faster channel variation, then the pilot distribution of the LTE can be presumed to perform better, and we will indeed show that Wimax suffers more from the effects of phase noise and Doppler with a worse degradation than the LTE system.

\section{OFDM SYSTEM}

The complex baseband equivalent of the OFDM transmission chain is depicted in Fig. 1. The $k$ th block of $M$ information symbols $\mathbf{a}_{k}=\left[a_{k}[0], \ldots, a_{k}[M-1]\right]$ is OFDM modulated by means of the inverse discrete Fourier transform (IDFT) over $M$ points.

1) Data and Pilot Format: In a frame of $N$ OFDM symbols, we assume that $M_{p}$ pilot subcarriers are scattered along some of the OFDM data symbols. When present, the pilots are considered equally spaced over the useful subcarriers [19], [20] at the indexes $i_{p}=\left(i_{o}+p D_{p}\right), p=0, \ldots, M_{p}-1$, where $D_{p}$ represents the pilot density, and $i_{o}$ is an offset to account for a possible shift in the pilot position, since the pilot position within the set of OFDM subcarriers can change along the frame, as in LTE. If a whole OFDM symbol is known, for example, as a preamble, the pilot density can be considered unitary for that OFDM symbol.

2) Channel and Phase Noise Model: After OFDM modulation, the signal is passed to the digital-to-analog converter (DAC) that gives the analog output for the up-conversion to $\mathrm{RF}$, which is then transmitted through the multipath radio channel.

The channel in the $k$ th OFDM symbol, after sampling at the receiver with sampling period $T$, is considered with an equivalent impulse response (including the effect of filters of $N_{\text {Ch }}$ taps $\mathbf{h}_{k}=\left[h_{k}[0], \ldots, h_{k}\left[N_{\mathrm{Ch}}-1\right]\right]$. We assume the use of a cyclic prefi with a length greater than the channel maximum delay $N_{\mathrm{Ch}}$, which is suitable to remove the intersymbol interference.

The power delay profil considered in the following results is the UMTS vehicular channel A [21].

We consider that the channel evolution is affected by a Doppler process characterized by a maximum spread frequency $f_{\text {max }}$ whose value, normalized to the OFDM symbol rate, is denoted by $f_{D}=f_{\max } M T$.

The phase noise $\theta(t)$ is introduced at the receiver, although it can account for both the term $\theta_{T}(t)$ introduced in the upconversion at the transmitter and the term $\theta_{R}(t)$ introduced in the down-conversion at the receiver side. The phase noise is assumed to evolve according to a Wiener random walk process [22], and the system performance in Section $\mathrm{V}$ is related to the phase noise increment variance $\sigma_{\theta}^{2}$ over the sample period $T$, where $\sigma_{\theta}^{2}=2 \pi B_{\theta} T$, with $B_{\theta}$ equal to the $3-\mathrm{dB}$ bandwidth of the Lorentzian power density carrier spectrum.

3) Received Signal: The sampled signal is demodulated by the discrete Fourier transform (DFT) to give the vector $\mathbf{X}_{k}=\left[x_{k}[0], \ldots, x_{k}[M-1]\right]^{T}$. The received signal at time $k$ in the $i$ th subcarrier, after cyclic prefi removal and OFDM demodulation, is

$$
x_{k}[i]=H_{k}[i] \Theta_{k}[0] a_{k}[i]+I_{k}[i]+W_{k}[i]
$$

where $H_{k}[i]$ is the complex channel coefficien for the subcarrier $i$ at time $k$ given by the DFT of the equivalent discrete-time channel impulse response

$$
H_{k}[i]=\sum_{n=0}^{M-1} h_{k}[n] e^{-j 2 \pi \frac{i n}{M}}, \quad i=0, \ldots, M-1
$$

and $I_{k}[i]$ is the ICI contribution, which originates from the loss of orthogonality due to phase noise

$$
I_{k}[i]=\sum_{\substack{n=0 \\ n \neq i}}^{M-1} a_{k}[n] H_{k}[n] \Theta_{k}[i-n]
$$

with

$$
\Theta_{k}[m]=\sum_{n=0}^{M-1} e^{j \theta_{k M+n}} e^{-j 2 \pi n \frac{m}{M}} .
$$

Note that the dc component of the phase noise DFT, i.e., $\Theta_{k}[0]=\sum_{n=0}^{M-1} e^{j \theta_{k M+n}}$, represents the CPE. In (1), $W_{k}[i]$ is 
the noise on subchannel $i$, which is given by

$$
W_{k}[i]=\sum_{n=0}^{M-1} w_{k}[n] e^{-j 2 \pi \frac{i n}{M}}, \quad i=0, \ldots, M-1
$$

where $w_{n}$ is the additive Gaussian noise at the receiver input (see Fig. 1) whose samples have variance $\sigma_{w}^{2}$, whereas the effect of phase noise on the additive noise samples is neglected due to the circular symmetry.

4) Matrix Formulation: The received signal can be written in matrix form, from (1), as

$$
\mathbf{X}_{k}=\mathbf{Q}_{k} \mathbf{a}_{k}+\mathbf{W}_{k}
$$

where $\mathbf{W}_{k}=\left[W_{k}[0], \ldots, W_{k}[M-1]\right]^{T}$ is the contribution of the additive noise, and the overall "channel" matrix $\mathbf{Q}_{k}$ comprises the effects of both the channel and the phase noise. The overall channel matrix $\mathbf{Q}_{k}$ is expressed in (7), shown at the bottom of the page, where we use the fact that the phase noise vector $\Theta_{k}=\left[\Theta_{k}[0], \ldots, \Theta_{k}[M-1]\right]^{T}$ is periodic with period $M$ due to the DFT operator.

The decision on the transmitted data is taken on the elements of the vector $\mathbf{Y}_{k}$, which is obtained from $\mathbf{X}_{k}$ by a proper equalization scheme, as described in the next section.

\section{RECEIVER SCHEMES}

According to the general signal formulation given by (1) and (6), we propose the following two schemes for detection and ICI cancellation:

- an ICI cancellation scheme on the whole OFDM symbol by inversion of $\mathbf{Q}_{k}$ (decorrelation);

- a partial ICI cancellation scheme by inversion of an approximation of $\mathbf{Q}_{k}$.

These methods will be compared with the per-subcarrier approach, where a one-tap equalizer, based on the MMSE criterion, is used [8], [10], [11].

\section{A. Per Subcarrier Equalization}

We firs recall the per-subcarrier approach [8], [10], [11], where equalization is obtained by the multiplication of each subcarrier by a coefficien $K_{k}[i]$, that is

$$
y_{k}[i]=K_{k}[i] x_{k}[i] \text {. }
$$

The optimum coefficien is derived according to the MMSE criterion, giving

$$
K_{k}[i]=\frac{Q_{k}^{*}(i, i)}{\left|Q_{k}(i, i)\right|^{2}+\sigma_{\mathrm{ICI}}^{2}+\sigma_{w}^{2}}
$$

where $(\cdot)^{*}$ denotes the complex conjugate, $Q_{k}(i, i)$ is the $i$ th diagonal element of the overall "channel" matrix $\mathbf{Q}_{k}$, and $\sigma_{\text {ICI }}^{2}$ represents the ICI variance. This approach corresponds to the consideration of the ICI as an additive noise term and removes the effect of the CPE only, which can be acceptable for a small phase noise and Doppler, which requires the estimation of only the diagonal terms in the matrix $\mathbf{Q}_{k}$.

\section{B. OFDM Symbol Equalization}

To cancel all the ICI components, we propose an equalization performed on the whole OFDM block. According to (6), the equivalent matrix to be inverted to equalize the received vector is $\mathbf{Q}_{k}$. Then

$$
\mathbf{Y}_{k}=\mathbf{Q}_{k}^{-1} \mathbf{X}_{k}
$$

This corresponds to a zero-forcing (ZF) equalization scheme and removes all the ICI components. However, since we consider different from zero all the elements of the matrix, this approach can lead to a high computational complexity in the determination of the inverse of the $M \times M$ matrix, since the size $M$ corresponds to the number of subcarriers and can be quite large for many systems, such as the considered standards LTE or IEEE 802.16.

\section{Partial ICI Reduction}

We propose a simpler equalization scheme, which achieves cancellation of the main ICI terms without the need of inverting the full matrix. The idea is based on the fact that the overall "channel" matrix $\mathbf{Q}_{k}$ can be seen as the convolution of the channel frequency response vector $\mathbf{H}_{k}=\left[H_{k}[0], \ldots\right.$, $\left.H_{k}[M-1]\right]^{T}$ and of the phase noise spectrum vector $\Theta_{k}$, corresponding to the fact that the received samples, before the receiver DFT, are multiplied by the phase noise. Noting that the phase noise is slowly varying with respect to the symbol rate, we can approximate the phase noise Fourier transform vector as different from zero only in a few terms around the dc component. This gives a band matrix with elements different from zero only in positions $(i, j)$ with $|i-j| \leq m$ around the main diagonal, and in particular, we can consider the case of a "tridiagonal" matrix, that is, with $m=1$, whose inversion can be performed with low-complexity algorithms.

Again, the vector sent to the decision element for data detection is obtained by (10).

\section{Channel Estimation}

The schemes presented before to compensate the channel and phase noise effects need the knowledge of the overall

$$
\mathbf{Q}_{k}=\left[\begin{array}{cclc}
H_{k}[0] \Theta_{k}[0] & H_{k}[1] \Theta_{k}[M-1] & \cdots & H_{k}[M-1] \Theta_{k}[M-1] \\
H_{k}[0] \Theta_{k}[1] & H_{k}[1] \Theta_{k}[0] & \cdots & H_{k}[M-1] \Theta_{k}[M-2] \\
\vdots & \vdots & & \vdots \\
H_{k}[0] \Theta_{k}[M-1] & H_{k}[1] \Theta_{k}[M-2] & \cdots & H_{k}[M-1] \Theta_{k}[0]
\end{array}\right]
$$


channel matrix $\mathbf{Q}_{k}$, or at least some of its elements. Then, in a practical implementation, $\mathbf{Q}_{k}$ in (9) and (10) is replaced by the estimated channel matrix, which will be denoted by $\tilde{\mathbf{Q}}_{k}$. To perform channel estimation, we employ a least squares (LS) approach, since it does not require the knowledge of the channel correlation matrix. On the other hand, the knowledge of some data symbols is required. Here, we consider a technique based on the following:

- the OFDM symbol used as frame preamble, if present (see Section II);

- the pilots scattered along the OFDM symbols;

- the detected data of past OFDM symbols, according to a decision-driven estimation.

We propose an estimation technique that combines the estimations provided by the pilots and obtained by the decisionfeedback technique.

Note that a fast update of the channel estimation is important not only in fast fading but also because of the time variability introduced by the phase-noise process.

\section{A. Pilot-Based Channel Estimation}

The LS criterion applied on the $M_{p}$ pilots gives a firs estimation of the diagonal terms of the matrix $\mathbf{Q}_{k}$ as

$$
\tilde{Q}_{k}\left(i_{p}, i_{p}\right)=\tilde{H}_{k}\left[i_{p}\right] \tilde{\Theta}_{k}[0]=\frac{x_{k}\left[i_{p}\right]}{a_{k}\left[i_{p}\right]}, \quad p=0, \ldots, M_{p}-1 .
$$

A frequency interpolation approach is then followed. The channel coefficient obtained on the pilots $i_{p}$ are extended to all adjacent subcarriers by the periodic sinc interpolating function

$$
\tilde{Q}_{k}(i, i)=\sum_{p=0}^{M_{p}-1} \tilde{Q}_{k}\left(i_{p}, i_{p}\right) \operatorname{sinc}_{\mathrm{p}}\left(\frac{i-i_{p}}{M / M_{p}}\right) .
$$

The choice of the periodic sinc function as an interpolation function is dictated by the fact that interpolation can be performed by a firs DFT over $M_{p}$ points, followed by zero padding up to $M$ points and by a subsequent IDFT over $M$ points, to have the interpolated channel for all subcarriers $i$.

Note that interpolation of the coefficient $\tilde{\mathbf{Q}}_{k}(i, i)$ is equivalent to the interpolation of the channel frequency response $H_{k}[i]$ due to the fact that all the terms are multiplied by the same coefficien $\Theta_{k}[0]$.

\section{B. Decision-Feedback Channel Estimation}

If we consider the channel not significantl varying over two symbols, the channel coefficient estimated by (11) can also be obtained from the previous OFDM symbol, exploiting the knowledge of the data provided by the detected symbols $\hat{a}_{k-1}[i]$, that is

$$
\tilde{Q}_{k-1}(i, i)=\tilde{H}_{k-1}[i] \tilde{\Theta}_{k-1}[0]=\frac{x_{k-1}[i]}{\hat{a}_{k-1}[i]} .
$$

This has the advantage of providing $M$ independent relations instead of $M_{p}$, so interpolation is not needed, but the disadvantage is that the estimated channel can already be "outdated" in the case of a fast Doppler effect.

Denoting with $\tilde{\mathbf{Q}}_{k}^{(p)}$ the estimate derived from the pilots and with $\tilde{\mathbf{Q}}_{k-1}^{(\mathrm{DF})}$ the estimate derived by decision feedback, the two are combined with a weighted linear combination, where the weight $0 \leq \beta \leq 1$ is optimized according to the Doppler and phase-noise conditions

$$
\tilde{\mathbf{Q}}_{k}=\beta \tilde{\mathbf{Q}}_{k}^{(p)}+(1-\beta) \tilde{\mathbf{Q}}_{k-1}^{(\mathrm{DF})} .
$$

In the second equalization scheme proposed, which requires the knowledge of all the matrix elements for its inversion, decoupling of the channel frequency response and phase noise vector is needed. First, the coefficient of the channel frequency response are obtained by

$$
\tilde{H}_{k}[i]=\frac{\tilde{Q}_{k}(i, i)}{\tilde{\Theta}_{k}[0]}
$$

where estimation of the CPE $\tilde{\Theta}_{k}[0]$ is obtained on the pilot subcarriers. Indeed, on the known pilot data symbols, by averaging the phase displacement with respect to the expected pilot symbol, one gets

$$
\tilde{\Theta}_{k}[0]=\frac{1}{M_{p}} \sum_{p=0}^{M_{p}-1} \arctan \left(\frac{x_{k}\left[i_{p}\right]}{\left|x_{k}\left[i_{p}\right]\right| a_{k}\left[i_{p}\right]}\right) .
$$

In other words, once the pilot symbol information $a_{k}\left[i_{p}\right]$ is removed, the phase deviation gives the estimated CPE. Then, the other elements of $\Theta_{k}$ are obtained by the LS criterion by solving the system [which directly derives from (6)]

$$
\mathbf{L} \Theta_{k}=\mathbf{X}_{k}
$$

where $\mathbf{L}$ is expressed as that in (18), shown at the bottom of the page. As can be seen, together with the channel coefficient $H_{k}[i]$, which are replaced by the estimate (15), the solution of the system of (17) requires the knowledge of the symbols $a_{k}[i]$ to derive the phase noise vector elements $\Theta_{k}[i]$. The data symbols are provided, according to a decision-feedback approach, by the detected data $\hat{a}_{k-1}[i]$. In other words, the

$$
\mathbf{L}=\left[\begin{array}{cccc}
H_{k}[1] a_{k}[1] & H_{k}[2] a_{k}[2] & \cdots & H_{k}[M-1] a_{k}[M-1] \\
H_{k}[2] a_{k}[2] & H_{k}[3] a_{k}[3] & \cdots & H_{k}[1] a_{k}[1] \\
\vdots & \vdots & & \vdots \\
H_{k}[M-1] a_{k}[M-1] & H_{k}[1] a_{k}[1] & \cdots & H_{k}[M-2] a_{k}[M-2]
\end{array}\right]
$$


estimated values are referred to the previous OFDM symbol $k-1$, and the phase-noise vector estimate $\tilde{\Theta}_{k-1}$ is the solution of the actual linear system

$$
\tilde{\mathbf{L}} \Theta_{k-1}=\mathbf{X}_{k-1}
$$

with $\tilde{\mathbf{L}}$ expressed as in (20), shown at the bottom of the page.

\section{Results}

As an example of the application, the 3GPP LTE parameters (set 1) are used in the following to obtain the error probability in detail:

- $M=512$ subcarriers;

- symbol rate $1 / T=7.68 \mathrm{MBd}$;

- useful subcarriers: 299;

- OFDM symbols per transmission time interval (TTI): 27;

- pilots $M_{p}=M / 4=128$ or $M / 8=64$ pilots per OFDM symbol.

Note that in both cases the number of pilots $M_{p}$ is greater than the length of the channel considered. The modulation format, if not otherwise stated, is quadrature phase shift keying (QPSK). With these parameters, we can consider that no OFDM symbol is available as preamble, whereas the pilot density is up to four (one subcarrier out of every four carries a pilot symbol), although the actual position of the pilots is not fi ed along the frame.

As another example of application, a complementary approach can be considered, where the OFDM symbols used as frame preamble can be even two, whereas the density of pilot subcarriers is lower. Here, the parameters suitable for the OFDM system specifie in the IEEE 802.16e-2005 Wimax standard [23] are considered, where some of the parameters are chosen (for example, the channel bandwidth) to be comparable with the UMTS LTE values, as follows:

- $M=256$ subcarriers;

- channel bandwidth: $7 \mathrm{MHz}$

- $M_{p}=8$ pilot subcarriers;

- preamble of one OFDM symbol;

- number of OFDM symbols per frame: 16 ;

- $M_{p}=8$ pilot subcarriers.

Considering the average number of pilots per OFDM symbol also in this case, the number of pilots is greater than the length of the channel.

\section{A. Per-Carrier (One-Tap) MMSE Equalization}

We firs consider the detection scheme where a simple equalization is implemented by the multiplication of a coefficien

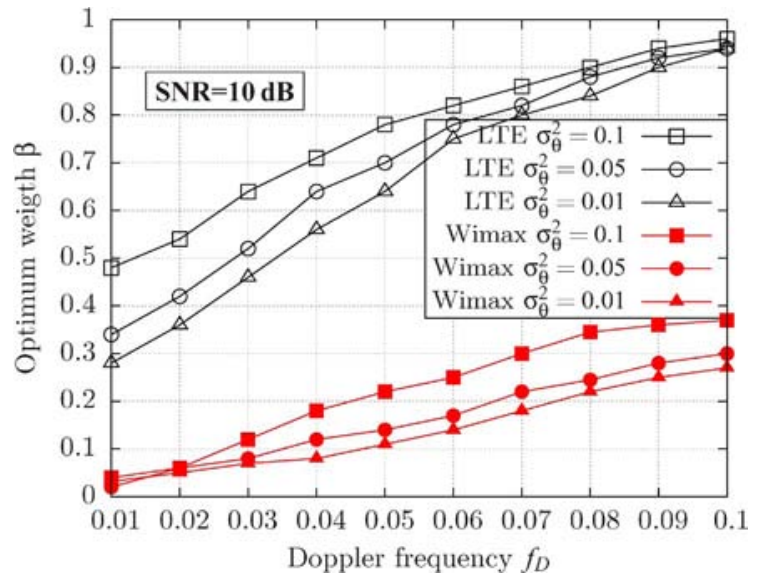

Fig. 2. Value of the optimum weight $\beta$ for LTE and Wimax with different conditions of phase noise, i.e., $\sigma_{\theta}^{2}=0.01, \sigma_{\theta}^{2}=0.05$, and $\sigma_{\theta}^{2}=0.1$.

per subcarrier, according to (9), and we present some results for the optimum value of the weight that is used to combine the pilot-based and DF estimates. In Fig. 2, the optimum value $\beta$ is plotted as a function of the Doppler frequency $f_{D}$ for different conditions on the phase noise with both LTE and Wimax scenarios while keeping a fi ed value of the SNR.

It can be seen that, as soon as the Doppler frequency $f_{D}$ increases, the optimum coefficien $\beta$ also increases as a consequence of the lower reliability of the past estimate with respect to the one derived from the pilots of the current OFDM symbol. On the other hand, if the pilot density is lower, as in the Wimax standard, the optimum value gets lower. Again, the effect of phase noise is comparable to a faster channel variation, thus determining a higher value of the optimum weight $\beta$. The sensitivity of the optimum value of $\beta$ on the SNR is not very relevant. In fact, although at higher values of SNR the reliability of the DF values is increasingly higher, the optimum $\beta$ is actually determined by the phase noise and the Doppler, which introduce variability in the channel and cause the fact that the DF estimate gets outdated as soon as the phase noise and the Doppler frequency become noticeable.

\section{B. ICI Cancellation by Matrix Inversion (Partial or Total)}

1) Slow Fading (Wimax and LTE): First, considering a channel with low mobility, characterized by a value of the Doppler frequency $\left(f_{D}=0.01\right)$, we can compare the choices of pilot and frame formats and their effect on the proposed ICI reduction and estimation algorithm. In Fig. 3, the penalty in the signal-to-interference-plus-noise ratio (SINR) is presented for the one-tap equalizer and for the partial matrix inversion, considering the matrix as tridiagonal, in different conditions of

$$
\tilde{\mathbf{L}}=\left[\begin{array}{ccc}
\tilde{H}_{k-1}[1] \hat{a}_{k-1}[1] & \cdots & \tilde{H}_{k-1}[M-1] \hat{a}_{k-1}[M-1] \\
\tilde{H}_{k-1}[2] \hat{a}_{k-1}[2] & \cdots & \tilde{H}_{k-1}[1] \hat{a}_{k-1}[1] \\
\vdots & & \vdots \\
\tilde{H}_{k-1}[M-1] \hat{a}_{k-1}[M-1] & \cdots & \tilde{H}_{k-1}[M-2] \hat{a}_{k-1}[M-2]
\end{array}\right]
$$




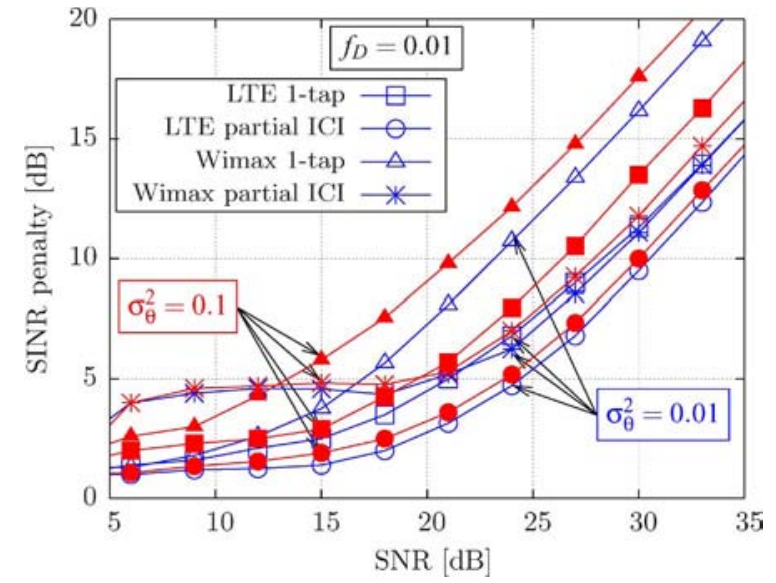

Fig. 3. SINR penalty with per-carrier equalization and partial matrix inversion for LTE and Wimax with Doppler frequency $f_{D}=0.01$ and phase noise $\sigma_{\theta}^{2}=$ 0.01 and $\sigma_{\theta}^{2}=0.1$

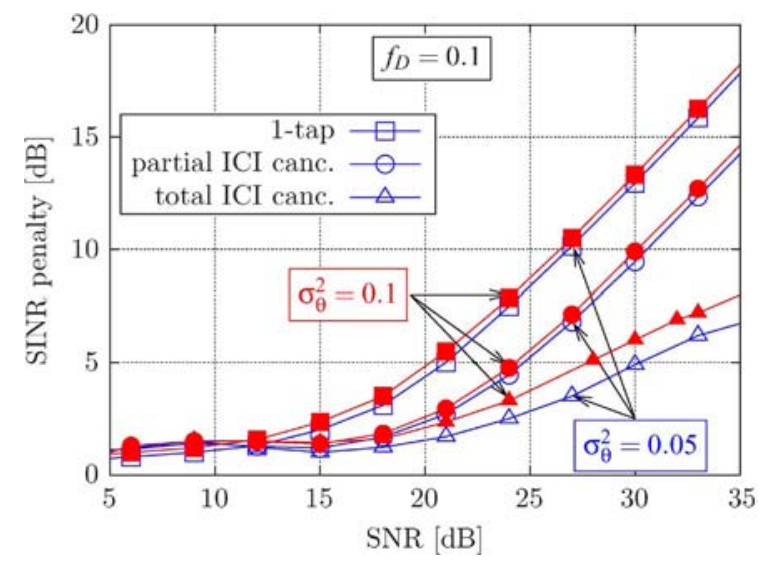

Fig. 4. SINR penalty with ICI cancellation by total or partial matrix inversion with Doppler frequency $f_{D}=0.1$ and two conditions of phase noise, i.e., $\sigma_{\theta}^{2}=0.05$ and $\sigma_{\theta}^{2}=0.1$.

phase noise, namely, $\sigma_{\theta}^{2}=0.01$ and $\sigma_{\theta}^{2}=0.1$, with both LTE and Wimax sets of parameters. The SINR penalty is define as the increase in the SNR value that is needed to achieve the same error probability that is obtained in the absence of phase noise and Doppler. Note that a linear increase of the penalty with respect to the SNR corresponds to a saturation effect in the error probability.

It can clearly be seen that a system like Wimax suffers more the effects of phase noise and Doppler, with a worse degradation than the corresponding LTE system. Note, however, that, although the one-tap equalizer shows a great degradation for increasing the phase noise, the partial ICI compensation is more robust.

2) Fast Fading (LTE): If we consider a fast-fading scenario characterized by the Doppler frequency $f_{D}=0.1$, the Wimax system suffers from the effect of the varying channel, so we present the following results only with the LTE parameters.

In Fig. 4, we present the effect of ICI cancellation that can be achieved by the inversion of the complete or partial matrix for a phase noise with $\sigma_{\theta}^{2}=0.05$ and $\sigma_{\theta}^{2}=0.1$ with Doppler frequency $f_{D}=0.1$.

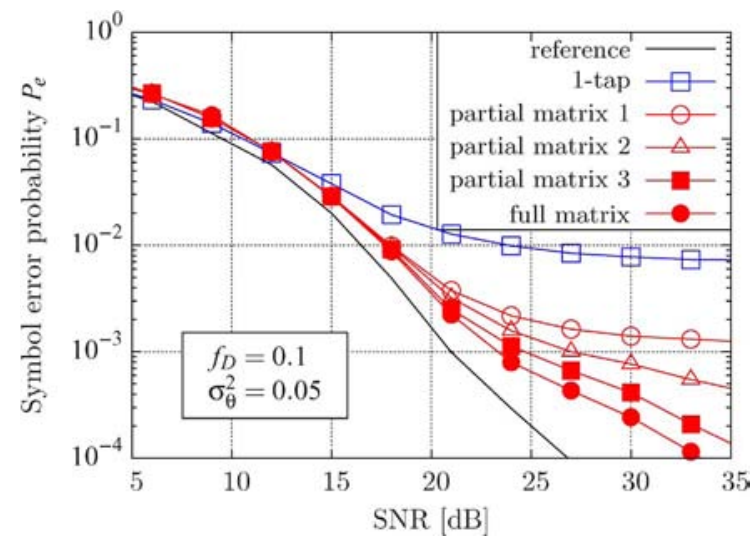

Fig. 5. Error probability with partial ICI matrix inversion, with Doppler frequency $f_{D}=0.1$, and $\sigma_{\theta}^{2}=0.05$.

The comparison among the values of the SINR penalty obtained by one-tap equalization and by ICI cancellation is presented in this figure The performance achieved by the simplifie ICI cancellation scheme is quite close to the performance provided by full matrix inversion. In both cases, the results are much better than the results achieved with one-tap equalization. Note that inversion of the total matrix does not lead to saturation in the error probability in the range of SNR values considered.

The closeness of the results in Fig. 4 obtained considering the whole matrix or its tridiagonal approximation suggests the use of the simplifie scheme, which requires a much lower computational complexity.

Its performance, now in terms of the uncoded symbol error probability, is shown in Fig. 5 for a high value of Doppler and phase noise, and compared with the one-tap equalizer. The different curves refer to the different numbers of terms considered around the diagonal in the estimation of the channel matrix from the tridiagonal matrix with one term around the diagonal to a band matrix with three terms on each side of the diagonal.

Although the partial ICI cancellation with just one term around the diagonal (tridiagonal matrix) can give a noticeable gain with respect to the one-tap equalizer, the error probability saturates to an error floo due to the residual ICI in the presence of high phase noise and Doppler, and a further gain is obtained by considering more terms in the channel matrix. On the other hand, almost the same performance as the full matrix inversion is achieved by considering the firs three terms.

To show the dependency of the channel estimation and ICI cancellation scheme on the pilot subcarrier density in fast fading, we present in Fig. 6 the uncoded symbol-error probability for different densities of pilot subcarriers, thus also varying the interpolation factor of the frequency estimation.

Reducing the number of pilot subcarriers from one out of every four to one out of every eight worsens a lot the estimation provided by pilots only (one-tap equalization), whereas the combined use of a DF estimation, as employed in the ICI cancellation schemes, suffers less from the pilot reduction.

The sensitivity of the proposed schemes to the modulation format is shown in Fig. 7, where the uncoded symbol-error probability is shown for QPSK and 16 quadrature-amplitude 


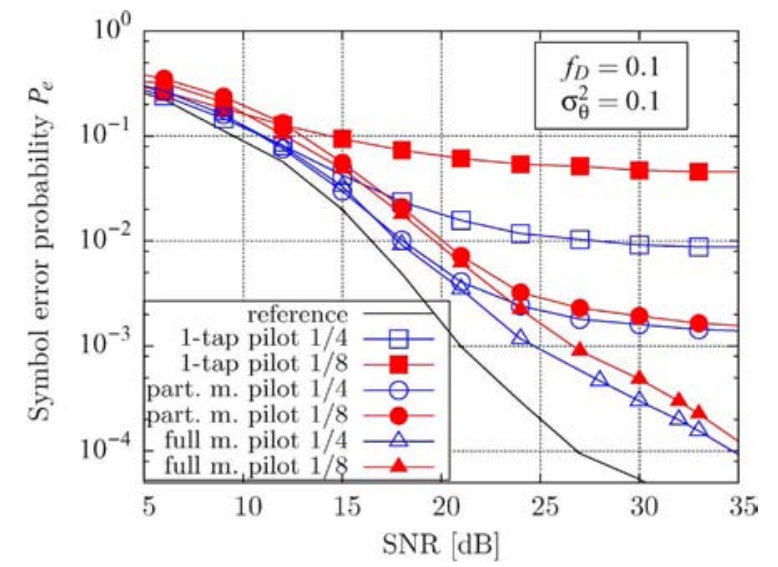

Fig. 6. Error probability for different receiver schemes with different pilot densities $(1 / 4$ or $1 / 8)$ with $f_{D}=0.1$ and $\sigma_{\theta}^{2}=0.1$.

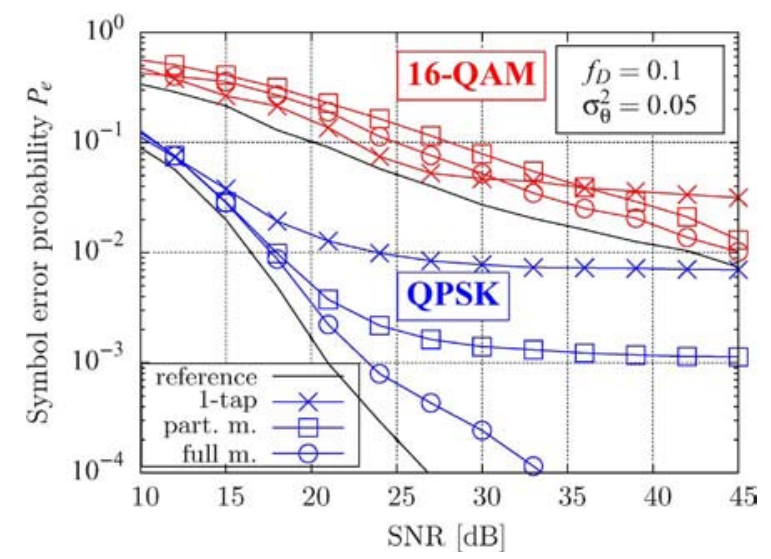

Fig. 7. Error probability for different receivers for QPSK and 16-QAM with $f_{D}=0.1$ and $\sigma_{\theta}^{2}=0.05$.

modulation (16-QAM). Together with the expected performance degradation as the constellation points increase, for 16-QAM modulation, we can appreciate a greater gain of the full matrix inversion with respect to the partial ICI reduction due to the worse ICI degradation arising in multilevel modulations also for lower values of SNR. Moreover, for very low values of SNR, we have a larger interval, where the one-tap per-carrier equalization performs slightly better than the ICI cancellation schemes due to the lower reliability of the estimate. On the other hand, by increasing the SNR, the partial or total cancellation schemes achieve a better performance.

\section{Comparison of the Proposed Schemes}

To compare the sensitivity of the proposed algorithms to the phase noise and to the Doppler spread frequency $f_{D}$, the penalty, that is, the SNR increase to achieve the error probability $P_{e}=10^{-2}$ (with respect to the ideal case without phase noise and Doppler), is plotted in Figs. 8 and 9.

First, in Fig. 8, the penalty is shown for a slow varying channel with the comparison between LTE and Wimax. The modulation format is QPSK.

It can be seen that for LTE, in up to high values of the phase noise, the penalty of the ICI cancellation schemes is quite

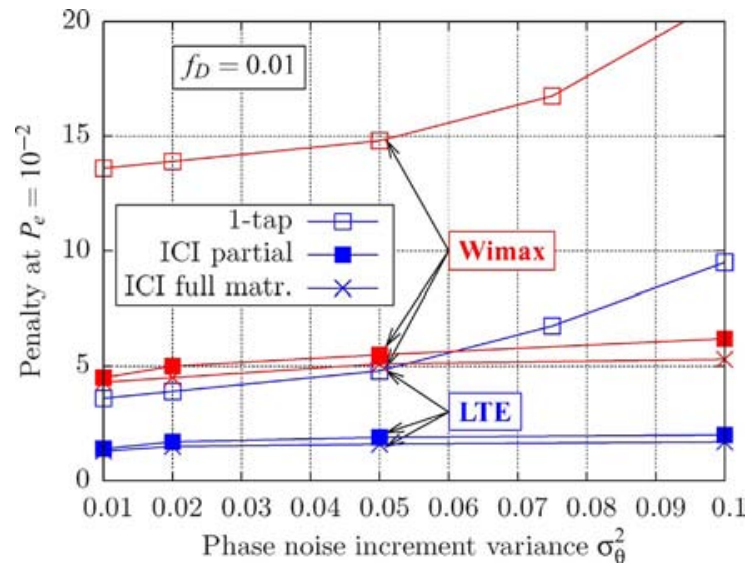

Fig. 8. Penalty at $P_{e}=10^{-2}$ as a function of $\sigma_{\theta}^{2}$ for $f_{D}=0.01$ for both the LTE and the Wimax set of parameters.

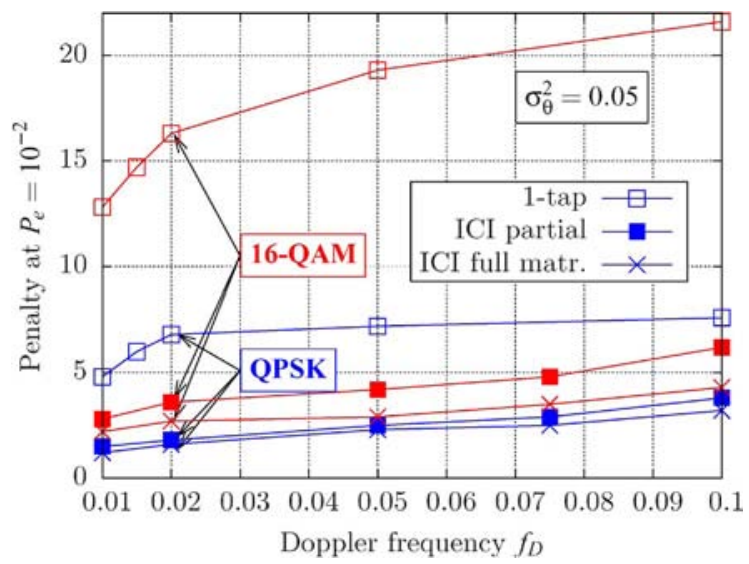

Fig. 9. Penalty at $P_{e}=10^{-2}$ as a function of $f_{D}$ for $\sigma_{\theta}^{2}=0.05$ for the LTE set of parameters.

low, and the reduced ICI canceller is almost equivalent to the complete ICI cancellation scheme. Note that the low sensitivity of the ICI cancellation schemes to the phase noise variance is also due to the fact that the penalty is evaluated for a low SNR at the error probability of $10^{-2}$. Indeed, much smaller values of the symbol error probability to perform the comparison cannot be considered since the presence of high phase noise produces a large degradation, giving a finit asymptotic $P_{e}$ for a large SNR (see Figs. 4 and 5). On the other hand, for Wimax, the penalty is much larger, although we can make the same considerations on the ICI cancellation schemes.

The results in Fig. 8 refer to a quite small value of the frequency $f_{D}$ representing the maximum Doppler spread. If we consider the sensitivity to the Doppler frequency $f_{D}$, the penalty is shown in Fig. 9 for a phase-noise increment variance $\sigma_{\theta}^{2}=0.05$ for the LTE set of parameters. Here, the case of 16-QAM is also considered as a comparison.

It can be seen that the penalty of the one-tap equalizer becomes almost insensitive to the Doppler frequency by optimizing the weight $\beta$ so that the estimation relies more on the current OFDM symbol under detection. The introduction of an ICI cancellation scheme based on a combined pilot and DF estimation allows the achievement of a low penalty, even in the presence of severe Doppler. The penalty of 16-QAM is 
much higher for the one-tap equalization scheme but becomes comparable with the case of QPSK if ICI cancellation is introduced, although it is referred to a higher value of SNR.

\section{CONCLUSION}

We have considered several ICI cancellation schemes, jointly with channel estimation, to compensate the channel distortion and the phase noise in OFDM systems with fast fading. We have seen that channel estimation implemented by a combination of a pilot-based scheme and a decision-feedback scheme can achieve good performance, together with an ICI cancellation by inversion of an equivalent overall channel matrix. Although the best performance is obtained by full matrix inversion, the proposed solution to consider only the firs terms of ICI, that is, a tridiagonal matrix, leads to results that are very close to the full matrix case, with a much lower complexity, which is suitable to also be employed in OFDM systems with a great number of subcarriers.

The pilot-based channel estimation requires a considerable, but realistic, number of pilot subcarriers to provide an updated channel to the equalization stage, particularly when the Doppler frequency is high.

\section{REFERENCES}

[1] J. Moon, J.-Y. Ko, and Y.-H. Lee, "A framework design for the nextgeneration radio access system," IEEE J. Sel. Areas Commun., vol. 24, no. 3, pp. 554-564, Mar. 2006.

[2] W. Songping and Y. Bar-Ness, "OFDM systems in the presence of phase noise: Consequences and solutions," IEEE Trans. on Comm., vol. 52, no. 11, pp. 1988-1996, Nov. 2004.

[3] L. Tomba, "On the effect of Wiener phase noise in OFDM systems," IEEE Trans. Commun., vol. 46, no. 5, pp. 580-583, May 1998.

[4] L. Tomba and W. A. Krzymien, "Sensitivity of the MC-CDMA access scheme to carrier phase noise and frequency offset," IEEE Trans. Veh. Technol., vol. 48, no. 5, pp. 1657-1665, Sep. 1999.

[5] A. García Armada and M. Calvo, "Phase noise and sub-carrier spacing effects on the performance of an OFDM communication system," IEEE Commun. Lett., vol. 2, no. 1, pp. 11-13, Jan. 1998.

[6] F. Munier, T. Eriksson, and A. Svensson, "Receiver algorithms for OFDM systems in phase noise and AWGN," in Proc. IEEE PIMRC, Sep. 2004, pp. 1998-2002.

[7] G. Liu and W. Zhu, "Compensation of phase noise in OFDM systems using an ICI reduction scheme," IEEE Trans. Broadcast., vol. 50, no. 4, pp. 399-407, Dec. 2004.

[8] S. Wu and Y. Bar-Ness, "Phase noise estimation and mitigation for OFDM systems," IEEE Trans. Wireless Commun., vol. 5, no. 12, pp. 3616-3625, Dec. 2006.

[9] D. Petrovic, W. Rave, and G. Fettweis, "Effects of phase noise on OFDM systems with and without PLL: Characterization and compensation," IEEE Trans. Commun., vol. 55, no. 8, pp. 1607-1616, Aug. 2007.

[10] G. Auer, A. Dammann, and S. Sand, "Channel estimation for OFDM systems with multiple transmit antennas by exploiting the properties of the discrete Fourier transform," in Proc. IEEE PIMRC, Sep. 7-10, 2003, vol. 2, pp. 1954-1958.

[11] P. Hammarberg and O. Edfors, "A comparison of DFT and SVD based channel estimation in MIMO OFDM systems," in Proc. IEEE PIMRC, Sep. 2006, pp. 1-5.

[12] D. Petrovic, W. Rave, and G. Fettweis, "Properties of the intercarrier interference due to phase noise in OFDM," in Proc. IEEE ICC, May 2005, pp. 2605-2610.
[13] Z. Jianhua and H. Rohling, "Analysis of ICI cancellation scheme systems with phase noise," IEEE Trans. Broadcast., vol. 50, no. 2, pp. 97-106, Jun. 2004.

[14] Y. Mostof and D. C. Cox, "ICI mitigation for pilot-aided OFDM mobile systems," IEEE Trans. Wireless Commun., vol. 4, no. 2, pp. 765-774, Mar. 2005.

[15] A. García Armada, "Estimation and correction of phase noise effects in orthogonal frequency division multiplexing," in Proc. IEEE GLOBECOM, San Francisco, CA, Nov. 2006, pp. 1-5.

[16] P. H. Moose, "A technique for orthogonal frequency division multiplexing frequency offset correction," IEEE Trans. Commun., vol. 42, no. 10, pp. 2908-2914, Oct. 1994.

[17] T. M. Schmidl and D. C. Cox, "Robust frequency and timing synchronization for OFDM," IEEE Trans. Commun., vol. 45, no. 12, pp. 1613-1621, Dec. 1997.

[18] M. Morelli and U. Mengali, "An improved frequency offset estimator for OFDM applications," IEEE Commun. Lett., vol. 3, no. 3, pp. 75-77, Mar. 1999.

[19] R. Negi and J. Cioffi "Pilot tone selection for channel estimation in a mobile OFDM system," IEEE Trans. Consum. Electron., vol. 44, no. 3, pp. 1122-1128, Aug. 1998.

[20] I. Barhumi, G. Leus, and M. Moonen, "Optimal training design for MIMO OFDM systems in mobile wireless channels," IEEE Trans. Signal Process., vol. 51, no. 6, pp. 1615-1624, Jun. 2003.

[21] Physical Channels and Mapping of Transport Channels onto Physical Channels, Mar. 2000. 3G TS 25.221, 3GPP TSG RAN WG1, Ver. 3.2.0.

[22] T. E. Parker, "Characteristics and sources of phase noise in stable oscillators," in Proc. 41st Annu. Freq. Control Symp., 1987, pp. 99-110.

[23] Air Interface for Fixed and Mobile Broadband Wireless Access Systems, IEEE Std. 802.16e-2005, Feb. 28, 2006.

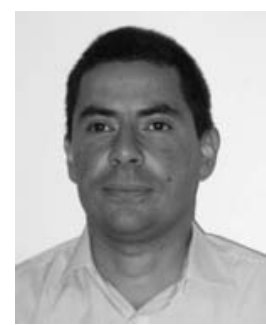

Roberto Corvaja (S'93-M'93) received the degree in electronic engineering and the Ph.D. degree in electronic engineering and telecommunications from the University of Padova, Padova, Italy, in 1990 and 1994, respectively.

Since 1994, he has been an Assistant Professor of telecommunications with the Department of Information Engineering, University of Padova. His research activity has involved coherent optical communications systems. Since 1995, his research activity has moved to wireless communication systems, in particular, to the analysis of the phase noise on different types of wireless communications. Between 1994 and 1995, he spent eight months with HewlettPackard Laboratories, Bristol, U.K., where worked on equalization algorithms for DECT. He took part in the European project ACTS-"CABSINET" on LMDS systems, as well as other research projects on wireless LAN and OFDM. His current main research activity concerns OFDM and MIMO-OFDM wireless systems.

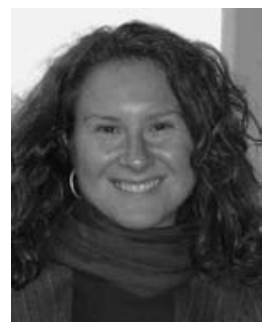

Ana García Armada (S'96-A'98-M'00-SM'08) received the Telecommunication Engineer degree from the Polytechnic University of Madrid, Madrid, Spain, in July 1994 and the Ph.D. degree in electrical engineering from the Polytechnic University of Madrid in February 1998.

She is currently an Associate Professor with the University Carlos III de Madrid, where she has occupied a variety of management positions. She has participated in several national and international research projects related to wireless communications. She is the coauthor of four books on wireless communications and signal processing. She has published 22 papers in international journals and more than 40 contributions to international conference proceedings. She has contributed to international organizations such as ITU and ETSI. Her main interests are OFDM and MIMO techniques and their application to wireless communications. 